\title{
Integrating nanophotonic concepts and topics into optics curricula
}

\section{Gregory Sonek}

Gregory J. Sonek, "Integrating nanophotonic concepts and topics into optics curricula," Proc. SPIE 9665, Tenth International Topical Meeting on Education and Training in Optics and Photonics, 96651B (3 June 2007); doi:

10.1117/12.2207535

SPIE Event: Tenth International Topical Meeting on Education and Training in Optics and Photonics, 2007, Ottawa, Ontario, Canada 


\title{
Integrating Nanophotonic Concepts and Topics into Optics Curricula
}

\author{
Gregory J. Sonek \\ Dept. of Electrical and Computer Engineering, Merrimack College, 315 Turnpike St., North Andover, MA 01845 \\ greg.sonek@merrimack.edu; 978-837-5000 x 4388
}

\begin{abstract}
Nanophotonics has emerged as a new and important field of study, not only in research, but also in undergraduate optics and photonics education and training. Beyond the study of classical and quantum optics, it is important for students to learn about how the flow of light can be manipulated on a nanoscale level, and used in applications such as telecommunications, imaging, and medicine. This paper reports on our work to integrate basic nanophotonic concepts and topics into existing optics and optical electronics courses, as well as independent study projects, at the undergraduate level. Through classroom lectures, topical readings, computer modeling exercises, and laboratory experiments, students are introduced to nanophotonic concepts subsequent to a study of physical and geometrical optics. A compare and contrast methodology is employed to help students identify similarities and differences that exist in the optical behavior of bulk and nanostructured media. Training is further developed through engineering design and simulation exercises that use advanced, vector-diffraction-based, modeling software for simulating the performance of various materials and structures. To date, the addition of a nanophotonics component to the optics curriculum has proven successful, been enthusiastically received by students, and should serve as a basis for further course development efforts that emphasize the combined capabilities of nanotechnology and photonics.
\end{abstract}

(C) 2007 Optical Society of America

OCIS codes: (000.2060) Education, (999.9999) Nanophotonics, (160.0160) Materials, (290.0270)

Scattering, (050.0050) Diffraction and gratings, (060.0060) Fiber optics, (300.0300) Spectroscopy.

\section{Introduction}

In recent years, education in nanotechnology [1] has accelerated, in part, due to the creation of programs such as the National Nanotechnology Initiative (NNI) that have promoted and supported research and development in this area. Nanophotonics [2,3], which combines the capabilities of nanoscience and technology with photonics [4], has emerged as a new and important field of study in its own right, encompassing the study of new optical interactions, materials, fabrication techniques, and architectures, including the exploration of natural and synthetic, or artificially engineered, structures [5] such as photonic crystals [6], holey fibers [7], quantum dots [8], sub-wavelength structures $[9,10]$, and plasmonics. With these developments come new and exciting opportunities for students at the undergraduate level to learn about how the flow of light can be manipulated on a nanoscale level, and used in critical applications that range from telecommunications and imaging, to medicine, healthcare, and environmental sensing. Such an exposure can provide breadth in the overall educational experience, as well as a heightened awareness of the pervasive role that this interdisciplinary field plays in today's society $[11,12]$. Herein, we report on our efforts to bring nanophotonics into the classroom by integrating basic concepts and topics into an existing optics curriculum.

\section{Background}

Many universities and colleges now have programs and centers dedicated to the pursuit of research and education in nanotechnology and nanophotonics. By comparison, Merrimack College, a small regional college with an Augustinian heritage that primarily serves the local Merrimack Valley communities of northern Massachusetts and southern New Hampshire, has only one course in optics within its Electrical Engineering curriculum. The Electrical Engineering Department, which has ABET accredited full- and part-time programs, has an enrollment of approximately 80 students, as well as a thriving Continuing Education program that draws part-time students from local industry, with primary employers in the military electronics, telecommunications, semiconductor, and instrumentation test and measurement markets. As part of its core curriculum, classes in electronics, VLSI, microprocessors, and senior project design provide students with extensive hands-on laboratory training, both in hardware, software, design, and test and measurement. There is also a keen interest within the college's Division of

Tenth International Topical Meeting on Education and Training in Optics and Photonics, edited by Marc Nantel, Proc. of SPIE Vol. 9665, 96651B - (c) 2007 SPIE, OSA, IEEE, ICO doi: $10.1117 / 12.2207535$ 
Science and Engineering to promote STEM (Science, Technology, Engineering, and Math) education [13] to nonscience and -engineering majors. The interdisciplinary nature of nanophotonics makes it an attractive candidate to facilitate this process, as well as to address the needs of local industry that have a need for well-trained students, and who utilize photonics in some form as part of their core business or product lines.

\section{Educational Goals and Objectives}

Based on the above, the challenge of bringing nanophotonics into the classroom at Merrimack College is one of taking a senior elective one-semester course entitled "Optical Electronics" and successfully integrating new concepts and topics into it that build upon existing course content, without initially creating an entirely new course. The course materials could, however, form the basis for a new course or multi-course sequence in the future. The overall goals of this effort are therefore threefold:

- Build upon existing course content in optoelectronics and photonics

- Leverage on-going faculty research activities in the field of nanophotonics

- Stimulate student self-discovery and exploration of nanophotonics through in- and out-of-class activities

At the end of the course, students are expected not only to have a working knowledge of basic photonic concepts, but also be able to articulate various aspects of nanophotonics from their classroom and experimental exercises. They should also have some idea about what approaches and tools can be used to design and engineer devices (or products) based on this technology. Seniors, and some advanced juniors, typically enter Optical Electronics with a solid background in fields and waves, but having prior sophomore-level physics classes as the only exposure to geometrical and wave optics.

Following the development of topics as presented in the text by Kasap [14], the course presents a detailed discussion of the wave nature of light (including polarization), and proceeds to develop the ideas of optical materials, light confinement and optical waveguiding, basic semiconductor principles, and the design and operation of semiconductor lasers and LEDs. It is within this framework that elements of nanotechnology and nanophotonics are introduced as extensions of existing course content.

The author's involvement in the design and development of specific nanophotonic structures and interactions provides a means to engage students and to show the relevance of the material to real-world devices and applications. Designs, fabrication methods, and test results on structures such as photonic crystal (PC) waveguides, sub-wavelength (SWS) anti-reflection structures, and quantum dots are presented to show photonic engineering in practice, rather than in abstract form. It is here that students acquire some hands-on training in lab experiments that involve the measurement of reflection, transmission, and polarization properties of various research-grade optical structures, including holographically-patterned SWS and PC structures.

Lastly, students are encouraged to explore the field of nanophotonics on their own through weekly readings and presentations of technical, trade journal, and web articles using, for example Photonics Spectra, Biophotonics, OPN, and Optics Express [15] as resources for topical materials that reinforce class concepts.

\section{General Course Structure and Development}

The challenge of presenting new concepts within a one-semester optical electronics course means that only a select number of topics can be covered. To this end, we have integrated nanophotonic concepts into a couple of key areas, those of which are outlined in Table I. These specific areas were chosen because they can be seamlessly integrated into the existing curriculum, are representative of key concepts, and can help students clearly identify the similarities and differences that exist between the optical behaviors of bulk and nanostructured media and devices. Such a compare- and contrast- methodology is used to help students distinguish nanoscale processes and interactions from those that occur on a much larger scale.

Beginning with a general introduction to photonics and its capabilities, the course quickly proceeds to a discussion of the wave nature of light and the scale of interactions, including light localization and cooperative electronic interactions. This is followed by the study of wave propagation and the optical properties of materials. It is here that ideas relating to the transmission, reflection, and coherent scattering by natural and artificially engineered materials, including quantum dot and periodic nanostructures like woodpiles and photonic crystals, are presented. The ability 
of these structures to confine and localize light are further addressed when the topic of optical waveguiding is introduced. Here, software simulation packages like BeamProp and GSolver can be used by students to design bulkand nano-scale devices, simulate wave propagation, and analyze optical performance by interpreting spectral transmittance and reflectance curves, or observing the propagation, confinement, and light localization effects within nanostructures.

TABLE I. Outline of topics integrating nanophotonics into an existing Optical Electronics course.

\begin{tabular}{|ll|}
\hline Photonics as an Enabling and Pervasive Technology \\
0 & Generation, transmission, detection, manipulation, and processing of light \\
0 & Role of nanophotonics within the photonics and optical electronics fields \\
- Wave Nature of Light and Scale of Interactions \\
0 & Characteristics of ray, wave, and quantum optics \\
0 & EM spectrum, size and scale, nanoscale interactions and light localization \\
- Wave Propagation and Optical Properties of Materials \\
0 & Refractive index, absorption, effective index, negative index \\
0 & Bulk and structured materials (dots, microspheres, woodpile, photonic crystal) \\
0 & Reflection, refraction, and Fresnel equations \\
0 & Interference, diffraction, and Bragg scattering as forms of coherent scattering \\
0 & Design and simulation of optical structures using BeamProp and GSolver \\
- & Light Confinement and Optical Waveguiding \\
0 & Properties of dielectric slab and optical fiber waveguides \\
0 & New waveguide types: photonic crystal, holey fiber, close-packed opal \\
0 & Refractive index profile, bandgap absorption, light localization \\
Semiconductor Lasers, LEDs, and Detectors \\
0 & Semiconductor statistics and energy band diagrams \\
0 & Modified density of states in dimensional structures \\
0 & Nanocavity lasers, thermal emitters, surface-enhanced emitters, IR detectors \\
Applications \\
0 & Spectroscopy of quantum dots and biomedical applications \\
0 & SWS structures for antireflection coatings, polarization control, and resonant reflection \\
0 & Photonic crystal devices for PICs, biosensing platforms, optical backplanes \\
\hline
\end{tabular}

The last two topics covered in the course include semiconductors, lasers and LEDs, and specific applications having nanophotonic solutions that are currently being addressed by industry. The section on semiconductors provides an opportunity to examine semiconductor statistics, band diagrams, and density of state functions in the case of bulk, thin film, periodic, and nanostructured media. These modified properties form the basis for discussion of new devices, as well as applications that range from the use of quantum dots in biomedical imaging and the design of antireflective and hydrophobic surfaces, to the development of photonic integrated circuits, high-speed optical backplanes, and biosensors, to name a few. The applications chosen for discussion tend to be focused on those that address semiconductor, telecommunication, or product engineering solutions, commensurate with the industries being served by the engineering program of the college and the interests of the part-time student body. 


\section{Examples and Illustrations}

Many of the concepts introduced throughout the course are illustrated using examples drawn from laboratory simulations, the fabrication and testing of real devices, or the results from current research and development activities in the field. An example of different nano-optical structures presented to students is shown in Fig. 1. These examples, which include quantum dots, holey fibers, and sub-wavelength structured surfaces (SWS) $[9,10]$ illustrate the diverse nature of structures and scales over which optical interactions can occur. Photonic structures in biology, such as the brittlestar arm, moth eye, or exoskeleton of the diatom Thalassia, are also nice examples that show how nature employs nanophotonics to manipulate the flow of light
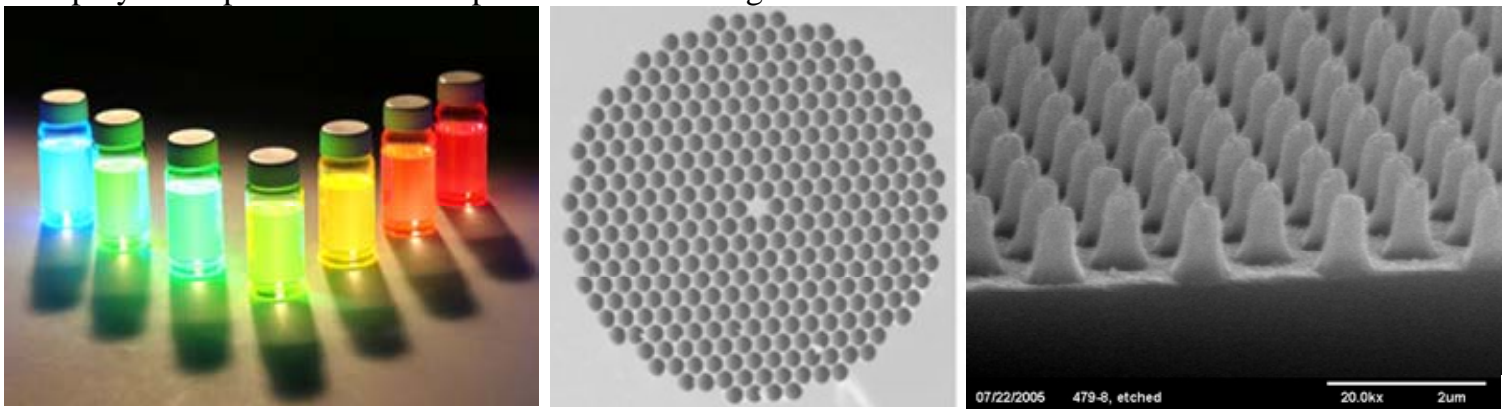

Fig. 1. Examples of nanophotonic materials and devices. These include quantum dots solutions (left), holey fibers (center), and diffractive sub-wavelength structures (right) designed and fabricated by the author.

Critical to any engineering course is design and simulation. Here, tools such as GSolver and BeamProp may be used to study the optical properties of different materials, geometries, and device configurations. An example of these tools and results that are demonstrated to students, and used by them in the class, are shown in Figs. 2 and 3. Fig. 2 shows one of the menus that is used in GSolver, a vector-diffraction software simulation tool, to enter optical material, wavelength, and geometric parameters prior to running a simulation of spectral transmittance and reflectance. Such a tool allows students to see the effects that parametric changes have on device performance.
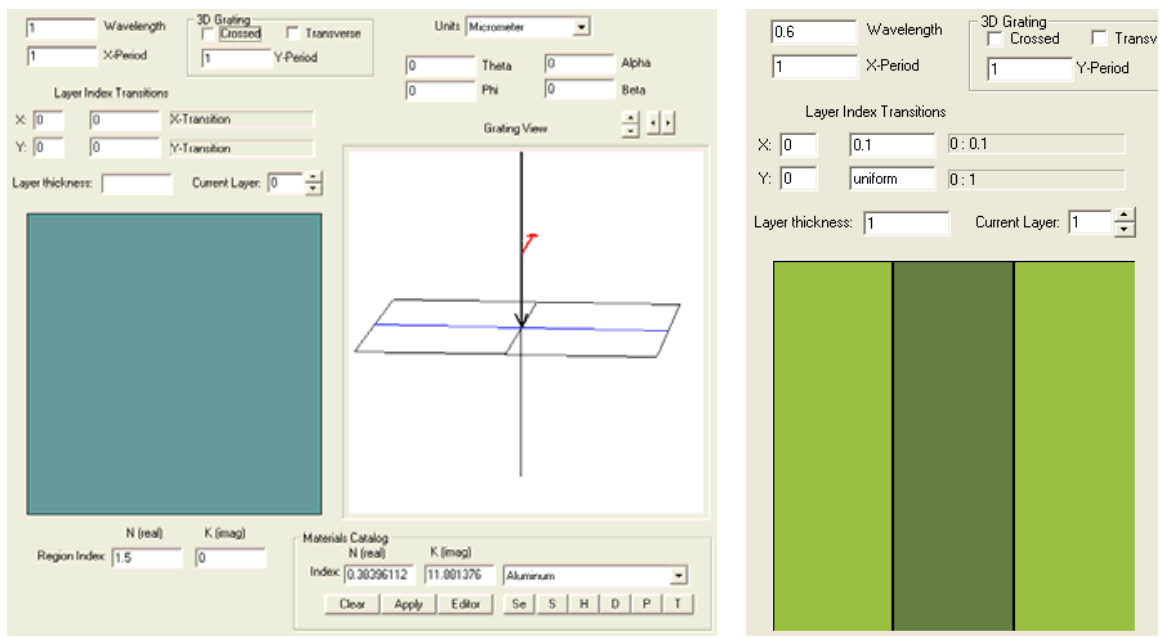

Fig. 2. GSOLVER editor (left) and Wavelength/Layer setting (right) menus used for simulating the optical properties of bulk and structured materials.

Optical waveguiding and the effects of light localization can be illustrated using a model for a photonic crystal slab waveguide, as shown in Fig. 3. These results, generated using finite-difference time domain techniques, show how an optical waveguide can be created using a linear array of air holes that have been patterned into a thin slab of silicon containing a periodic array of smaller-sized air holes. The example also provides a basis for the discussion of coherent scattering, including the dramatic visual and optical effects it can produce. 


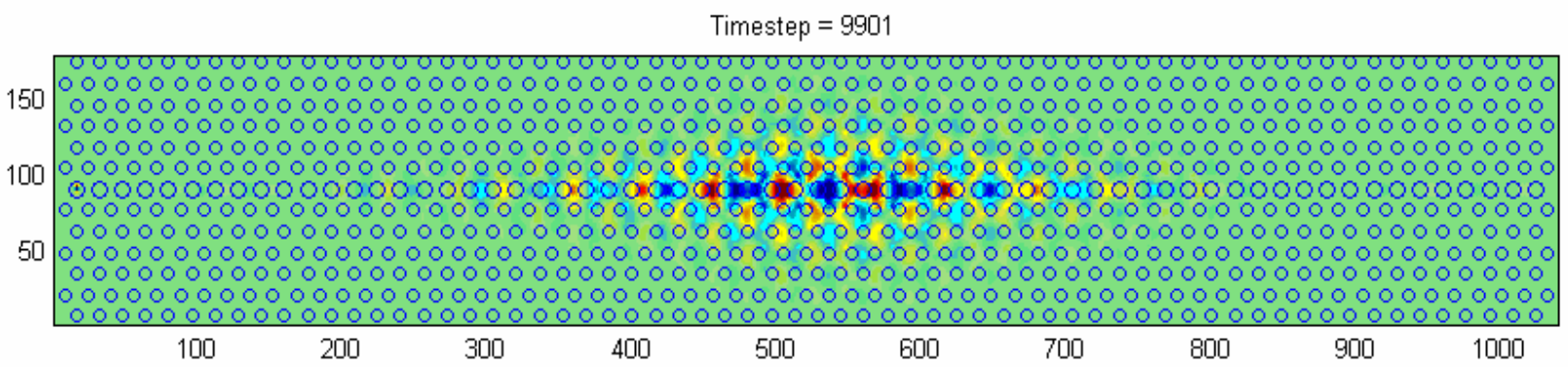

Fig. 3. Wave propagation in a slab photonic crystal waveguide. This example illustrates the concepts of optical waveguiding, light localization, and the process of coherent scattering in a photonic nanostructure.

No engineering class would be complete without showing the results from some real devices or products, or by giving students the opportunity to make sample measurements for themselves. For this purpose, an Ocean Optics high-resolution spectrometer is used to make spectral measurements in the visible wavelength range, while the services of a local vendor are used for FTIR measurements in the near- to mid-infrared spectral range. An example of the latter is shown in Fig. 4 for a silicon-based SWS structure that was designed and fabricated by the author to operate as an antireflection (AR) coating in the $2-6 \mu \mathrm{m}$ spectral range. This result emphasizes how the effective index properties of photonic nanostructures can be used to manipulate the spectral and polarization properties of light.

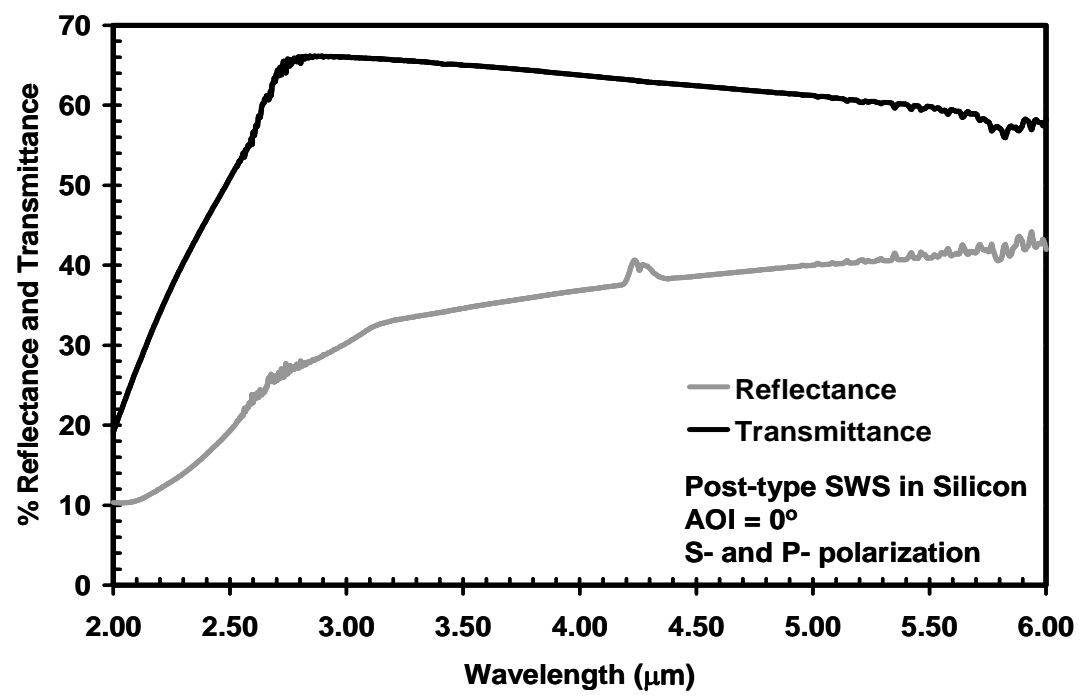

Fig. 4. Reflectance and transmittance spectra for a two-dimensional SWS structure designed for broadband antireflection (AR) operation in the $2-6 \mu \mathrm{m}$ MWIR wavelength range.

An example that demonstrates the use of a photonic nanostructure in a critical application is that of the chemical or molecular biosensor, as shown in Fig. 5. Here, a resonant reflecting structure is used as a biosensing platform for sensitive protein detection. Though this device has yet to be realized in our lab, it provides students with an opportunity to consider various design requirements, material choices, and the possible advantages of exploiting nanoscale optical interactions for the high resolution detection of molecules that, themselves, are nanoscale in dimension.

Lastly, to foster the continued discovery and exploration of nanophotonics outside of the classroom, students are encouraged, and required, to find, read, and present, articles found in the various technical and trade journals. The cover pages of several such trade magazines are shown in Fig. 6. These magazines are most instrumental in showing students the relevance of their studies in optical electronics, and the new developments that are rapidly emerging in the nanophotonics field. 


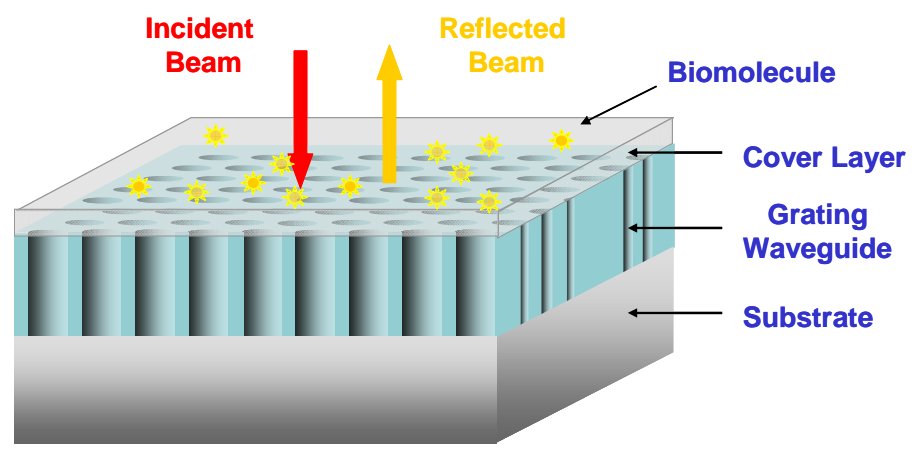

Fig. 5. Resonant reflecting structure used to illustrate the research and development of a new biosensing platform for protein detection using nanophotonic structures.
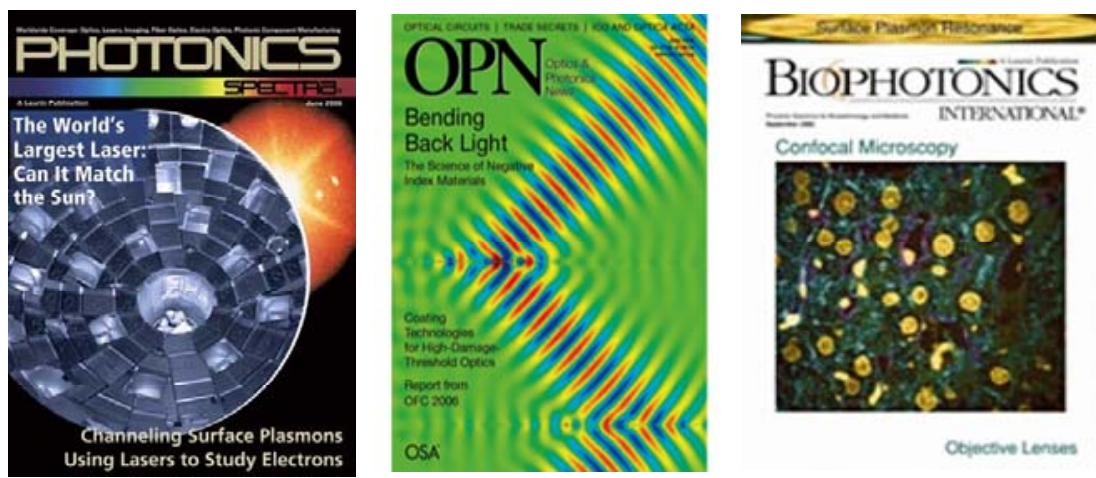

Fig. 6. Cover pages from the journals of Photonics, OPN, and Biophotonics. These magazines are used to get students engaged and excited about their studies in optical electronics and nanophotonics.

\section{Conclusion}

Nanophotonics is rapidly becoming a pervasive technology, with applications that range from optical and fiber optic communications to imaging, medicine, and healthcare. In this paper, we have described an approach for introducing nanophotonics to undergraduate students in a small liberal arts college engineering program, and bringing these basic concepts into the classroom. Through trade-journal and on-line readings, weekly discussions, and a limited number of laboratory experiments, students are able to learn about nanophotonics and its many facets, including optical interactions, materials, fabrication techniques, and uses. While limited in scope, this work should serve as a basis for further course development efforts that emphasize the capabilities of nanotechnology and photonics combined.

\section{Acknowledgements}

This research was funded, in part, by a Murray Faculty Fellowship, sponsored by the Paul E. Murray Fund at Merrimack College, and an educational grant from the Ocean Optics Corporation (Dunedin, FL).

\section{References}

[1] C.P. Poole and F.J. Owens, Introduction to Nanotechnology, (Wiley-Interscience, NY 2003). See also E.L. Wolf, Nanophysics and Nanotechnology (Wiley-VCH 2006); and, National Nanotechnology Initiative (http://nano.gov).

[2] P.N. Prasad, Nanophotonics (Wiley-Interscience, 2004).

[3] H. Rigneault, J.M. Lourtioz, C. Delalande, and J.A. Levenson, eds., Nanophotonics (ISTE Publishing, 2006).

[4] B.E.A. Saleh and M.C. teach, Fundamentals of Photonics (Wiley Interscience, NY 1991).

[5] P. Vukusic and J. R. Sambles, "Photonic structures in nature," Nature 424, 852 (2003).

[6] J.D. Joannopoulos, R.D. Meade, and J. N. Winn , Photonic Crystals: Molding the Flow of Light (Princeton University Press, 1995).

[7] A. Bjarklev, J. Broeng, and A.S. Bjarklev, Photonic Crystal Fibres (Springer, Berlin 2001)

[8] G.W. Bryant and G.S. Solomon, eds., Optics of Quantum Dots and Wires (Artech House Publishers, NY 2004).

[9] D.H. Raguin, S. Norton, and G.M. Morris, "Subwavelength structured surfaces and their applications," in Critical Reviews of Optical Science and Technology, Proc. SPIE Vol. CR49, 234 - 261 (1993). 
[10] P. LaLanne and M. Hutley, "The optical properties of artificial media structured at a subwavelength scale," in Encyclopedia of Optical Engineering, J. Steed, ed., (Dekker Publishing, USA, 2003) pp. 62 - 71.

[11] Harnessing Light: Optical Science and Engineering for the 21 $1^{\text {st }}$ Century, Committee on Optical Science and Engineering, National Research Council (National Academy Press 1998).

[12] M.S. Unlu, M.F. Ruane, B.B. Goldberg, T.D. Moustakas, B.E. Saleh, and M.C. Teich, "PRIDE: Photonics Research in Interdisciplinary Education,” Proc. IEEE Lasers and Electro-Optics Society 1996 Annual Meeting, No. 1626, June 1996.

[13] Transforming Undergraduate Education in Science, Mathematics, Engineering, and Technology, Committee on Undergraduate Science Education, Center for Science, Mathematics, and Engineering Education, National Research Council (National Academy Press, Washington, D.C. 1999).

[14] S.O. Kasap, Optoelectronics and Photonics: Principles and Practices (Prentice Hall, NJ 2001).

[15] Photonics Spectra and Biophotonics International (Laurin Publishing, Pittsfield, MA) (www.photonics.com); Optics and Photonics News and Optics Express (Optical Society of America, Washington DC) (www.osa.org). 